\title{
Disorder-induced vibrational anomalies from crystalline to amorphous solids
}

\author{
Ling Zhang, ${ }^{1,2}$ Yinqiao Wang, ${ }^{2}$ Yangrui Chen $\odot,{ }^{2}$ Jin Shang, ${ }^{2}$ Aile Sun, ${ }^{2}$ Xulai Sun, ${ }^{2}$ Shuchang Yu, ${ }^{2}$ Jie Zheng, \\ Yujie Wang, ${ }^{2}$ Walter Schirmacher, ${ }^{3}$ and Jie Zhang $\circledast^{2,4, *}$ \\ ${ }^{1}$ School of Automation, Central South University, Changsha 410083, China \\ ${ }^{2}$ School of Physics and Astronomy, Shanghai Jiao Tong University, Shanghai 200240, China \\ ${ }^{3}$ Institut für Physik, Universität Mainz, Staudinger Weg 7, D-55099 Mainz, Germany \\ ${ }^{4}$ Institute of Natural Sciences, Shanghai Jiao Tong University, Shanghai 200240, China
}

(Received 27 April 2021; accepted 6 September 2021; published 16 September 2021)

\begin{abstract}
The origin of the boson peak - an excess of the density of states over Debye's model in glassy solids-is still under intense debate, among which some theories and experiments suggest that the boson peak is related to a van-Hove singularity. Here we show that the boson peak and the van-Hove singularity are well separated entities by measuring the vibrational density of states of a two-dimensional granular system, where the packings are tuned gradually from a crystalline to polycrystalline structure and toward an amorphous material. We observe the coexistence of the boson peak and the van-Hove singularities being well separated in the polycrystals. The van-Hove singularities gradually shift to higher-frequency values while broadening their shape. They disappear completely when the structural disorder becomes sufficiently high. By analyzing the system at different degrees of disorder, we find that the boson peak is associated with spatially uncorrelated random flucutations of the shear modulus, whereas the smearing of the van-Hove singularities is associated with spatially correlated fluctuations of the shear modulus.
\end{abstract}

DOI: 10.1103/PhysRevResearch.3.L032067

Glassy materials show anomalies with respect to Debye's predictions, for example, a prominent peak in the specific heat relative to $T^{3}$ [1-3], a plateau in the temperature variation of the thermal conductivity [4,5], and a remarkable peak in the reduced density of states $D(\omega) / \omega^{d-1}$, normalized with respect to the Debye law, with $d$ being the dimension [6-9]. This latter peak is called "boson peak" (BP) and is closely related to the mentioned other thermal anomalies. The BP is associated with a strong increase of the sound attenuation $[10,11]$ as well as a minimum in the frequency-dependent sound velocity $[10,11]$. Near the BP frequency, the sound waves reach the Ioffe-Regel limit, where the mean free path of the sound wave approaches its wavelength.

To understand the origin of the BP-related vibrational anomalies has been taken as a challenge by many workers ever since their discoveries [1,6-8,12-17]. In most efforts, the structural disorder has been considered crucial. The influence of disorder on vibrational spectrum has been theoretically modelled by spatially fluctuating potentials [18-20], force constants $[8,21,22]$, and elastic constants $[5,11,23]$. In particular, excellent agreement between theory and both experiments and simulations has been achieved using effective-medium theories $[11,24,25]$.

\footnotetext{
*jiezhang2012@sjtu.edu.cn

Published by the American Physical Society under the terms of the Creative Commons Attribution 4.0 International license. Further distribution of this work must maintain attribution to the author(s) and the published article's title, journal citation, and DOI.
}

However, there still exists a controversial and opposite opinion, that the BP would be a washed-out version of the lowest van-Hove singularity (VHS) of the transverse phonon branch [22,26-28]. The argument in favor of this interpretation is that in lattice models of force-constant disorder the VHS peak smoothly transforms into the boson peak with increasing disorder $[8,22,28]$ and that the BP in some glasses appears in the same frequency regime as the VHS peak of the corresponding crystal, if rescaled with the Debye frequency. A rather strong argument against this interpretation is that near and beyond the BP frequency the Ioffe-Regel limit shall be reached and a dispersion of the transverse branch cannot be defined $[9,29]$.

To shed new light on this important issue, it is desirable to present more evidence, in particular from experiments. Granular materials show striking similarities with glasses. If prepared properly, then jammed granular matter can have a disordered structure like a liquid and can be mechanically stable like a solid. Moreover, granular materials can show caging dynamics and dynamical heterogeneity as well [30-33]. Recently, these similarities have been placed in a more rigorous theoretical framework where the jamming line of granular materials lies deep within the Gardner phase of the hardsphere glass $[34,35]$.

From the theoretical perspective, the density of states (DOS), constitutes the essence in understanding the thermal anomalies of the low-temperature physics of glasses, i.e., the BP, since the temperature dependence is encapsulated into the separate Bose factor. Therefore, much of the physical insight of the BP can be gained by analyzing the DOS of the zero-temperature amorphous packing, 
A
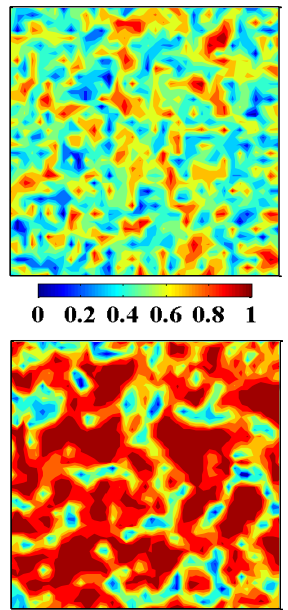

$\mathbf{E}$

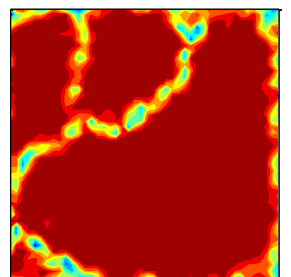

a

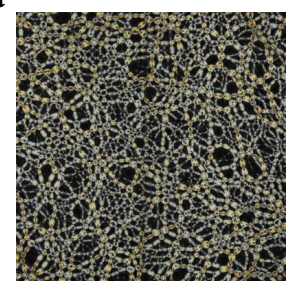

c

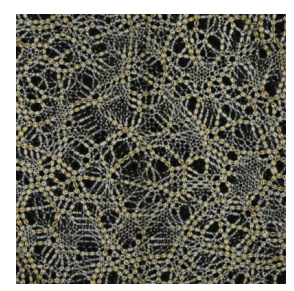

e

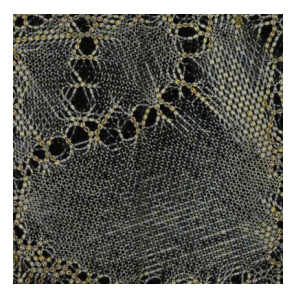

B

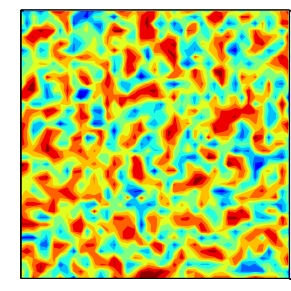

D

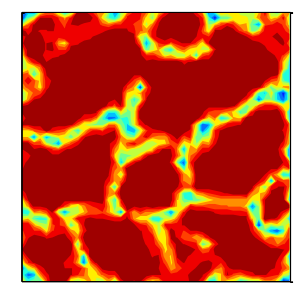

$\mathbf{F}$

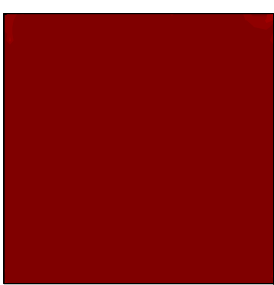

b

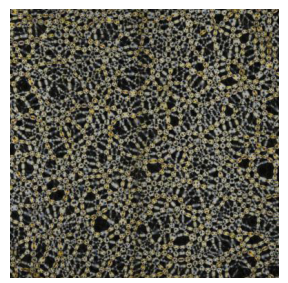

d

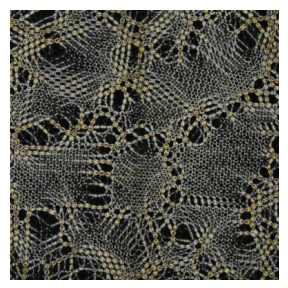

f

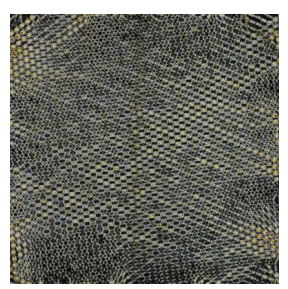

FIG. 1. Images of the bond orientation parameter $\psi_{6}^{i}[(\mathrm{~A})-(\mathrm{F})]$ and stresses [(a)-(f)] of packings of various degrees of disorder, showing force chains, i.e., those filament-like structures composed of particles carrying large stresses [49,50]. Note that the same letters in upper and lower cases denote the same packing, and the color bar under panel (A) indicates the values of $\psi_{6}^{i}$. The ratio $R$ between the number of small and large disks and the disorder parameter $\eta$ are [(A) and (a)] $R=1.0, \eta=1.0 ;[(\mathrm{B})$ and (b) $R=6.25, \eta=0.74 ;[(\mathrm{C})$ and (c) $] R=33.1$, $\eta=0.41 ;[(\mathrm{D})$ and $(\mathrm{d})] R=178.92, \eta=0.20 ;[(\mathrm{E})$ and $(\mathrm{e})]$ (polycrystal): $R=1.0, \eta=0.13 ;[(\mathrm{F})$ and (f)] (granular crystal): $R=1.0, \eta=0.0$.

in particular the anomalous vibrational properties of granular materials [15-17,36,37]. Using a two-dimensional (2D) granular packing is more advantageous than $3 \mathrm{D}$ systems, especially experimentally, since contact forces can be precisely measured to construct the Hessian matrix [29,32,38], despite that the precise form of the excess DOS, i.e., the DOS subtracting the Debye's DOS [39], in 2D is still under intense debate in contrast to that of $3 \mathrm{D}$ systems where the excess DOS has a robust scaling same as that of the sound attenuation [17,23,29,39-44].

Here we present evidence to demonstrate the separateness of the BP and the VHS by means of a 2D experimental system of granular materials in which the structural order can be tuned from the limit of crystal to glass. We find that BP and VHS are clearly separated. Specifically, we observe the coexistence of the BP and VHS in polycrystals of moderate degrees of disorder. Strikingly, we also observe that when the degree of disorder increases, the VHS shifts to higher frequencies. These observations strongly suggest that in general there is no generic connection between the BP and the lowest VHS, in contrast to the propositions in the literature [22,26,27]. In addition, we investigate the relationship between the BP and the relative fluctuations of the shear modulus $\delta G /\langle G\rangle$ and the degree of structural disorder. We find that the boson peak is associated with spatially uncorrelated random flucutations of the shear modulus $\delta G /\langle G\rangle$, whereas the smearing of the van-Hove singularities is associated with spatially correlated fluctuations of the shear modulus $\delta G /\langle G\rangle$ that are reminiscent of a crystalline solid. The smeared VHS disappears completely at large disorder.

In this experiment, we study the vibrational properties of $2 \mathrm{D}$ dense packings of photoelastic disks with various degrees and types of structural disorder, ranging from a glassy system to a granular crystal with spatially fluctuating contact forces and force constants [45-48]. This is achieved by systematically changing the number ratio of small disks (diameter $1.0 \mathrm{~cm})$ to large disks $(1.4 \mathrm{~cm})$. For a ratio of $1: 1$, we obtain the maximum random packing, whereas in the pure small-disk system the packing forms a crystal of a triangular lattice. We use the bond-orientation parameter $\psi_{6}^{i}$ to determine the local hexagonal order, as depicted in Figs. 1(A)-1(F). The $\psi_{6}^{i}$ is defined as $\psi_{6}^{i}=\left|\frac{1}{N_{\mathrm{nn}}} \sum_{k}^{N_{\mathrm{nn}}} e^{6 j \theta_{i k}}\right|$ [51-53], where $N_{\mathrm{nn}}$ is the number of nearest neighbors, $\theta_{i k}$ is the bond angle between disk $i$ and disk $k$, and $j \equiv \sqrt{-1}$. The average $\psi_{6}^{i}$ varies between the values of $\left\langle\psi_{6}^{i}\right\rangle=0.552$ (for a ratio $1: 1$ ) and $\left\langle\psi_{6}^{i}\right\rangle=$ 1 (for the granular $\operatorname{crystal}^{1}$ ). In samples of intermediate composition of small and large disks, $\left\langle\psi_{6}^{i}\right\rangle$ varies continuously. We define a disorder parameter $\eta=\left(1-\left\langle\psi_{6}^{i}\right\rangle\right) / 0.448$, such that $\eta=0$ for granular crystal and $\eta=1$ for the maximum random packing (see Fig. S1 in the Supplemental Materials).

\footnotetext{
${ }^{1}$ By "granular crystal" we mean a structural single crystal, which, however, exhibits force-constant disorder and contact-force disorder.
} 

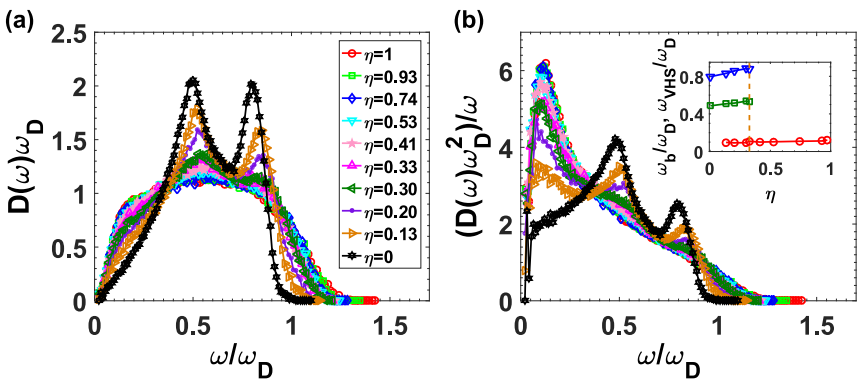

FIG. 2. The DOS's (a) and reduced DOS's (b) of packings of different disorder parameters $\eta$. Here each curve is ensemble averaged over 10 independent packings of the same $\eta$. Moreover, the frequency $\omega$ is normalized by the Debye frequency $\omega_{D}$. Inset of (b): The positions of the boson peak $\omega_{b} / \omega_{D}$ (red $\circ$ ), the first and second VHS $-\omega_{\mathrm{vH} 1} / \omega_{D}$ (green $\square$ ) and $\omega_{\mathrm{vH} 2} \omega_{D}$ (blue $\nabla$ ) vs $\eta$. The dashed line denotes the minimum $\eta$ beyond which the two VHS disappear. Here $p=26.5 \pm 0.2 \mathrm{~N} / \mathrm{m}$.

For a given $\eta$, we repeat the experiment 10 times, starting from an independently prepared initial packing. Images of the spatial distribution of $\psi_{6}^{i}$ and stress images of six different $\eta$ are shown in Fig. 1, including the polycrystals in Figs. 1(C) and 1(c), Figs. 1(D) and 1(d), and Figs. 1(E) and 1(e) and a crystalline packing with weak disorder of contact forces in Figs. 1(F) and 1(f) [45-47]. To create an initial packing, we use a biaxial-compression or shear device (biax) [29,32,54], where four walls of a square domain move inwards symmetrically while its center is fixed. This biax is filled with photoelastic disks, from $\sim 1500$ to $\sim 2200$ depending on $\eta$. Starting from an unjammed state, we compress the system isotropically until it approaches a granular solid state of various packing fractions depending on the value of $\eta$, e.g., its volume fraction $\phi \sim 0.84$ if $\eta=1$ [55] and $\phi \sim$ 0.90 if $\eta=0$. During the compression, we constantly apply agitations to eliminate residual stress to produce a random close packing (RCP) of bidisperse particles of $\eta=1$ (size ratio $1: 1.4$ and a number ratio $1: 1$ ), resembling the corresponding RCP of frictionless particles $[15,56]$. We prepare the initial packing of other $\eta$ in a similar way. Starting from an initial packing, we compress the system into a set of highly jammed packings in a series of steps, where the strain increment in each step is $4.26 \times 10^{-4}$. At each step, images of the particle configurations and stress images are taken for further analyses. After a precise measurement of the contact forces between the disks [29,32,54], we construct the Hessian matrices to analyze the corresponding normal modes of the set of different packing [29,32]. Using the Hessian matrices, we then determine the DOS and reduced DOS of these systems [29,32].

The DOS and the reduced DOS of packings of different $\eta$ are shown in Fig. 2, where we normalize the frequency $\omega$ using Debye's frequency $\omega_{D}-\mathrm{a}$ natural frequency unit, and we let $\int D\left(\frac{\omega}{\omega_{D}}\right) d\left(\frac{\omega}{\omega_{D}}\right)=1$. When $0<\eta \leqslant 0.33$, on each curve, a shoulder in the DOS or a corresponding BP appears in the reduced DOS in the low-frequency regime, and, meanwhile, two VHS's appear in the high-frequency regime, as seen in Figs. 2(a) and 2(b). When $\eta>0.33$, two VHS's barely exist with two small bumps reminiscent of VHS's, as shown
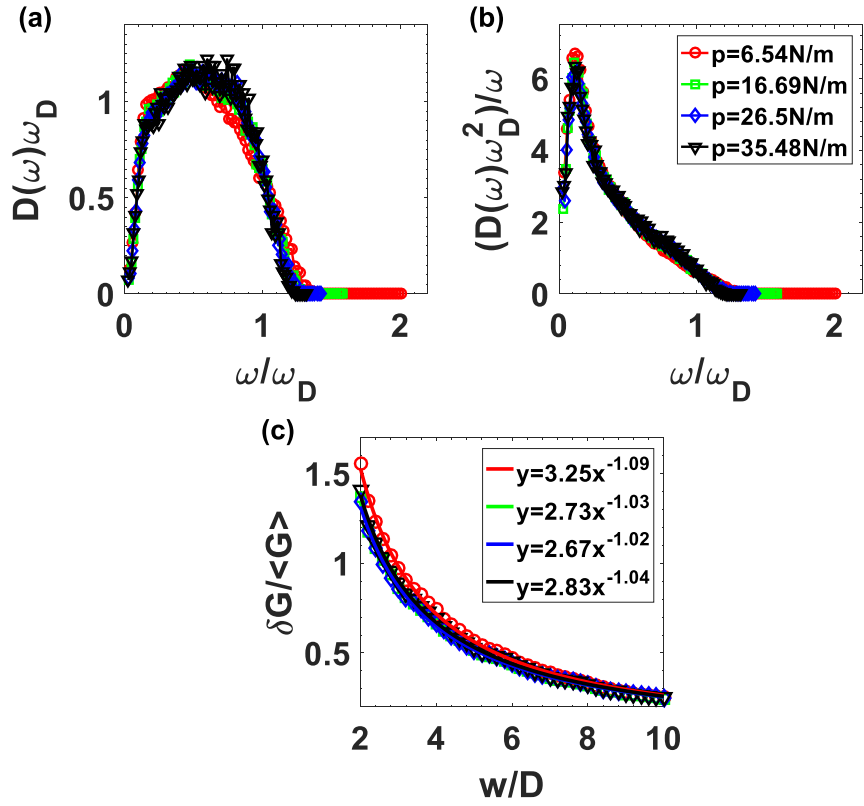

FIG. 3. The DOS (a) and the reduced DOS (b) at different $p$ collapse for the strongly disordered system $(\eta=1)$. Only data points from four different values of the pressure $p$ are shown for clarity, results at other $p$ are similar. (c) The relative fluctuations of the shear modulus, $\delta G /\langle G\rangle$ vs $w / D$ at different pressure for the strongly disordered system $(\eta=1)$, which is corresponding to (a) and (b). The results are independent of $p$. Note that here $w / D$ represents the rescaled course-grain size, where $D$ is the weighted average diameter of disks, i.e., $\frac{N_{b} D_{b}+N_{s} D_{s}}{N_{b}+N_{s}}$, here $N_{b}\left(N_{s}\right)$ indicates the number of the larger (smaller) disks for the whole system, and $D_{b}\left(D_{s}\right)$ indicates the diameter of the larger (smaller) disks.

in Figs. 2(a) and 2(b). The evolution in Fig. 2 shows a clear level repulsion due to the gradual loss of symmetry going from the crystalline structure to a more and more disordered system. This appears to push the states of two VHS's toward the low-frequency regime (leading to the formation of the BP) and simultaneously toward the high-frequency regime (causing Anderson localization [8,38,57-59]), in agreement with theoretical findings $[8,60,61]$.

In addition, a striking characteristic is that two VHS's shift gradually to higher frequencies as $\eta$ increases, as shown in Fig. 2(b) and its inset. The above observations are in sharp contrast to Ref. [22] where the lowest VHS gradually shifts to lower frequencies as the force-constant disorder increases, leading to the conclusion [22] that the BP would be generically related to the lowest VHS. Moreover, the frequency of the lowest VHS and the BP frequency are close with a ratio of $\sim 1.4-2$ for sufficiently high force-constant disorder [22], whereas in our case these two frequencies are rather distinct with a ratio of $\sim 5$ as shown in Fig. 2(b). The opposite trends in the change of VHS may be understood from the different nature of disorder between Ref. [22] and our system: Reference [22] is a lattice model with the force-constant disorder, different from the structure disorder in our system. These two different types of disorder have long been believed to be equivalent [22] but have qualitative differences in causing the evolution of the VHS, as confirmed in the above discovery. 

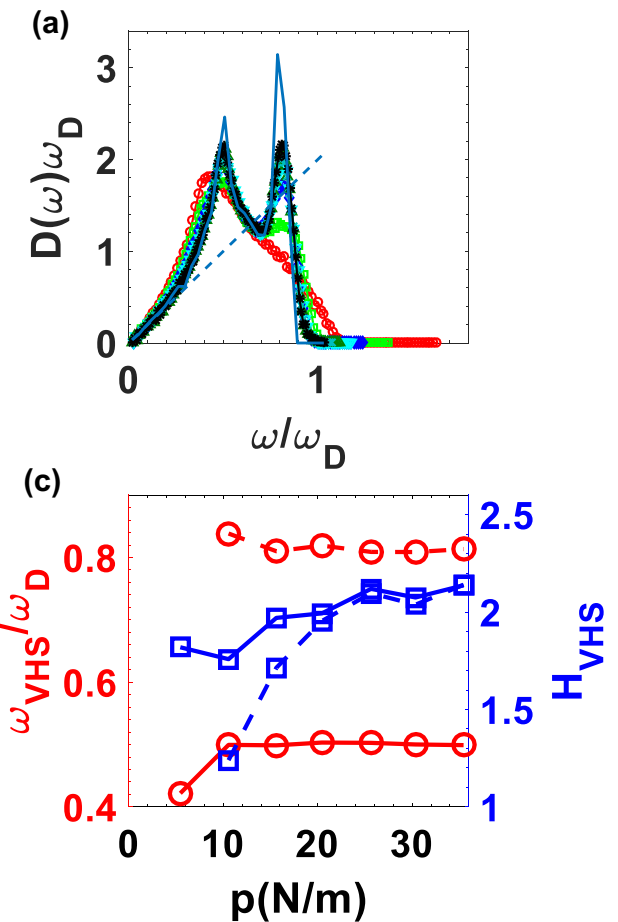

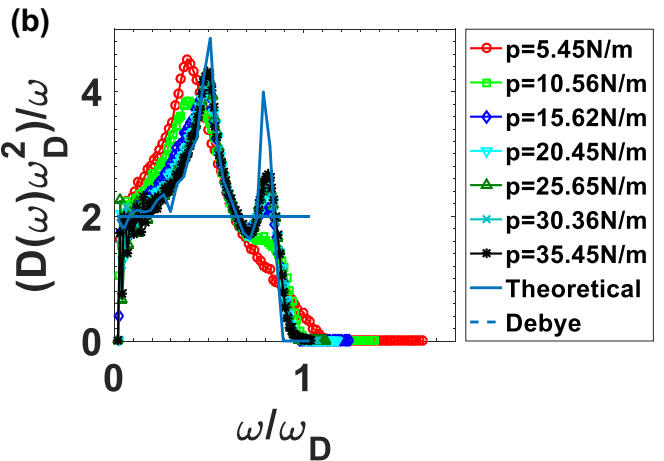

(d)

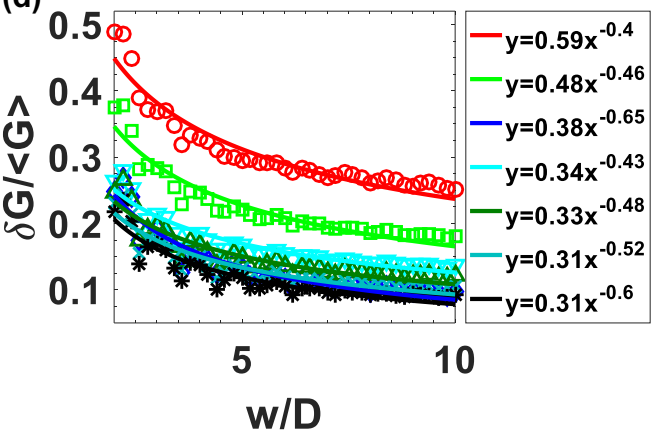

FIG. 4. The DOS (a) and the reduced DOS (b) rescaled by the Debye frequency $\omega_{D}$ at different pressure for the granular crystals $(\eta=0)$. Each curve in (a) and (b) is ensemble averaged over 10 different runs at the same pressure. (c) With double $y$-axes: The left $y$ axis represents the frequencies of two VHS's $\left[\omega_{\mathrm{vH} 1}\right.$ (red $\circ$, solid line) and $\omega_{\mathrm{vH} 2}$ (red $\circ$, dashed line)] and the right $y$ axis represents the heights of two VHS's [ $H_{\mathrm{vH} 1}$ (blue $\square$, solid line) and $H_{\mathrm{vH} 2}$ (blue $\square$, dashed line)] versus pressure. Note that the data of the second VHS at $p=5.45 \mathrm{~N} / \mathrm{m}$ are not shown here, due to the disappearance of the second VHS. (d) The relative fluctuations of shear modulus, $\delta G /\langle G\rangle$ vs $w / D$, at different pressure corresponding to (a) and (b). The results are dependent on $p$. Note that here $w / D$ represents the rescaled course-grain size, where $D$ is the weighted average diameter of disks. Each curve in (a)-(d) is ensemble averaged over 10 different runs at the same pressure.

In the simulations of Tong et al. [62,63], the authors also observed the coexistence of the BP and the VHS's in a weakly disordered 2D system, by systematically increasing the structural disorder starting from a perfect crystal. However, there are two differences between their findings and our experimental results, as follows. First, in the simulations, it is easy to isolate disorder type one at a time by exploring force-constant disorder on a perfec lattice $[62,63]$ and lattice disorder [63], polydispersity of particles [62], the fluctuations of local coordination number, or vacancies [63] to sufficiently high degrees. In our experiment, the simultaneous presence of the different types of disorder considered in Refs. [62,63] is inevitable. Moreover, the positions of the lowest VHS hardly change with disorder $[62,63]$, while in our findings, the lowest VHS evidently shifts to higher frequencies, as seen in Fig. 2, which further verifies the distinction between BP and VHS.

Apparently, here BP's are necessarily induced by disorder, including both the structural disorder and the force-constant disorder. To disentangle the two, we first investigate the role of the force-constant disorder in the strongly disordered system $(\eta=1)$ and the granular crystals $(\eta=0)$. Here we consider the relative fluctuations of shear modulus, $\delta G /\langle G\rangle$, which is confirmed to be the leading contribution to BP formation $[11,32,64]$ (see the Supplemental Materials for the calculations of the local moduli [65]).

As shown in Figs. 3(a) and 3(b), the normalized DOS's and the reduced DOS's do not show significant changes in the strongly disordered systems $(\eta=1)$ when the pressure $p$ changes by a factor of five. The measured $\delta G /\langle G\rangle \propto$ $w^{-1.0 \pm 0.05}$ at different pressure show little pressure dependence, as depicted in Fig. 3(c), in consistent with the results in Figs. 3(a) and 3(b). Here the $\delta G /\langle G\rangle \propto w^{-1.0 \pm 0.05}$ scaling can be understood based on the central limit theorem and the lack of long-range correlations in the spatial fluctuations of moduli, equivalent to Gaussian statistics of the modulus flucutation $[11,32,64]$. (See also Fig. S2- Fig. S4 for more details on the spatial correaltion and the probability distributions in the Supplemental Materials [65].)

Figure 4(a) shows the normalized DOS's at different pressure $p$ in the granular crystals, where the triangular-lattice structure is fixed but the force constant is disordered, due to the force network and the nonlinear interactions [47,48]: When $p \leqslant 5.45 \mathrm{~N} / \mathrm{m}$, only the transverse VHS is left followed by a knee structure that gradually transforms to the longitudinal VHS when $p>5.45 \mathrm{~N} / \mathrm{m}$. Correspondingly in Fig. 4(b), only the transverse VHS is followed by a knee structure in the reduced DOS's, nearly invisible at $p=5.45$ $\mathrm{N} / \mathrm{m}$. In contrast, the transverse VHS changes little after normalizing $\omega$ by $\omega_{D}$, which further verifies that the BP and the VHS are two different entities. Additionally, when $p>15.62$ $\mathrm{N} / \mathrm{m}$, both DOS and reduced DOS nearly collapse, as shown in Figs. 4(a) and 4(b). Figure 4(c) shows the changes of both frequencies and heights of the two VHS's with pressure, eventually leveling off. Here the change of the DOS's with pressure shows that the granular crystal is not an ordinary crystal, with the force inhomogeneities and force-constant 
disorder at contacts due to the nonlinear contact-force law.

To understand the results in Figs. 4(a)-4(c), we also measure $\delta G /\langle G\rangle$, as shown in Fig. 4(d). Apparently, there is a change in $\delta G /\langle G\rangle$ when $p \leqslant 15.62 \mathrm{~N} / \mathrm{m}$, in consistent with the evolution of the DOS's and reduced DOS's, especially the longitudinal VHS. When $p>15.62 \mathrm{~N} / \mathrm{m}, \delta G /\langle G\rangle$ does not depend on $p$ any more, consistent with the behavior of the DOS's and reduced DOS's. Here $\delta G /\langle G\rangle$ has a much smaller value at the particle scale compared to that of Fig. 3(c), and moreover $\delta G /\langle G\rangle \propto w^{-0.54 \pm 0.05}$ decays much slower with spatial correlations-reminiscent of the crystalline structure.

Furthermore, the shear modulus fluctuations are at least five times as large as the fluctuations of the bulk modulus $\delta B /\langle B\rangle$ (see Fig. S4 in the Supplemental Materials [65]). Recall that our previous work [32] shows that in the $\eta=1.0$ systems the wavelength of the transverse wave at the BP frequency is comparable to the characteristic length associated with the shear modulus fluctuations and a strong spatial correlation exists between the nonaffine shear modulus and low-frequency modes. Hence, the BP and the VHS's are well-separated entities with different natures of shear modulus fluctuations: The BP formation is strongly associated with Gaussian random fluctuations of the shear modulus, whereas the evolutions of the VHS's are related to the spatially correlated fluctuations of the shear modulus.

The systematic change of the exponent $\alpha$ with $\eta$ in the scaling of $\delta G /\langle G\rangle \propto w^{-\alpha}$ can be found in Fig. S5(g) in the Supplemental Materials [65], showing that the Gaussian random fluctuations of shear modulus start as early as $\eta \sim 0.33$ with $\alpha \approx 1.0$ for $\eta \geqslant 0.33$ and interestingly the two VHS's disappear around $\eta \sim 0.33$, as shown in Fig. 2. Additionally, the spatial fluctuations of shear modulus suggest that the formation of the BP is closely related to the local soft regimes in space, in good agreement with the early propositions $[9,11,14,32]$. Moreover, the relative height of the BP shows a nontrivial scaling function of $\delta G /\langle G\rangle \eta$, for which we do not have an theoretical explanation. [See Fig. S5(g) in the Supplemental Materials [65] for more details.]

In conclusion, we have studied the vibrational properties of dense granular materials with a broad range of structural disorder and find that the BP and the VHS are two well-separated features. We observe the coexistence of the BP and the VHS in packings of moderate degrees of disorder with $\eta \leqslant 0.33$ and the complete disappearance of the two VHS's when $\eta>0.33$. Around $\eta=0.33$, there is an associated qualitatively difference in the spatial flucutions of shear modulus.

By analyzing the fluctuations of the shear modulus, $\delta G /\langle G\rangle$, at different pressure $p$ both in the strongly disordered system $(\eta=1)$ and the granular crystals $(\eta=0)$, we find the Gaussian random spatial fluctuations of $\delta G /\langle G\rangle$ play a crucial role in the formation of the BP [11,24,29,32].

We further find that the formation of the BP is closely related to local soft regimes of negative shear modulus, as found in packings of all $\eta$ except in the granular crystals. Moreover, we find that $\delta G /\langle G\rangle$ fluctuate randomly in space as Gaussian random variables when $\eta>0.33$, below which its fluctuation has spatial correlation and two VHS's become visible. Hence, the formation of the BP is accompanied with spatially uncorrelated random fluctuations of the shear modulus and the evolution of the VHS are accompanied with spatially correlated fluctuations of the shear modulus.

This work is supported by the National Natural Science Foundation of China (NSFC) under Grants No. 11774221 and No. 11974238. This work is also supported by the Innovation Program of Shanghai Municipal Education Commission under Grant No. 2021-01-07-00-02-E00138. L.Z. also thanks the support by NSFC under Grant No.11904410 and by Natural Science Foundation of Hunan Province under Grant No. 2021JJ40712. We also acknowledge the support from the student innovation center of Shanghai Jiao Tong University.
[1] R. C. Zeller and R. O. Pohl, Thermal conductivity and specific heat of noncrystalline solids, Phys. Rev. B 4, 2029 (1971).

[2] C. C. Yu and A. J. Leggett, Low temperature properties of amorphous materials: Through a glass darkly, Comments Condens. Matter Phys. 14, 231 (1988).

[3] A. P. Sokolov, R. Calemczuk, B. Salce, A. Kisliuk, D. Quitmann, and E. Duval, Low-Temperature Anomalies in Strong and Fragile Glass Formers, Phys. Rev. Lett. 78, 2405 (1997).

[4] J. J. Freeman and A. C. Anderson, Thermal conductivity of amorphous solids, Phys. Rev. B 34, 5684 (1986).

[5] W. Schirmacher, Thermal conductivity of glassy materials and the "boson peak", Europhys. Lett. 73, 892 (2006).

[6] U. Buchenau, M. Prager, N. Nücker, A. J. Dianoux, N. Ahmad, and W. A. Phillips, Low-frequency modes in vitreous silica, Phys. Rev. B 34, 5665 (1986).

[7] V. K. Malinovsky and A. P. Sokolov, The nature of boson peak in Raman scattering in glasses, Solid State Commun. 57, 757 (1986).
[8] W. Schirmacher, G. Diezemann, and C. Ganter, Harmonic Vibrational Excitations in Disordered Solids and the "Boson Beak", Phys. Rev. Lett. 81, 136 (1998).

[9] H. Shintani and H. Tanaka, Universal link between the boson peak and transverse phonons in glass, Nat. Mater. 7, 870 (2008).

[10] G. Monaco and S. Mossa, Anomalous properties of the acoustic excitations in glasses on the mesoscopic length scale, Proc. Natl. Acad. Sci. USA 106, 16907 (2009).

[11] A. Marruzzo, W. Schirmacher, A. Fratalocchi, and G. Ruocco, Heterogeneous shear elasticity of glasses: the origin of the boson peak, Sci. Rep. 3, 1407 (2013).

[12] J. Jäckle, in Amorphous Solids: Low-Temperature Properties, edited by W. A. Phillips (Springer, Berlin, 1981), p. 135.

[13] U. Buchenau, N. Nücker, and A. J. Dianoux, Neutron Scattering Study of the Low-Frequency Vibrations in Vitreous Silica, Phys. Rev. Lett. 53, 2316 (1984).

[14] B. B. Laird and H. R. Schober, Localized Low-Frequency Vibrational Modes in a Simple Model Glass, Phys. Rev. Lett. 66, 636 (1991). 
[15] L. E. Silbert, A. J. Liu, and S. R. Nagel, Vibrations and Diverging Length Scales Near the Unjamming Transition, Phys. Rev. Lett. 95, 098301 (2005).

[16] L. E. Silbert, A. J. Liu, and S. R. Nagel, Normal modes in model jammed systems in three dimensions, Phys. Rev. E 79, 021308 (2009).

[17] H. Mizuno, H. Shiba, and A. Ikeda, Continuum limit of the vibrational properties of amorphous solids, Proc. Natl. Acad. Sci. USA 114, E9767 (2017).

[18] V. G. Karpov, I. Klinger, and F. N. Ignatiev, Theory of the lowtemperature anomalies in the thermal properties of amorphous structures, Zh. eksp. teor. Fiz. 84, 760 (1983) [Sov. Phys. JETP 57, 439 (1983)].

[19] U. Buchenau, Y. M. Galperin, V. L. Gurevich, and H. R. Schober, Anharmonic potentials and vibrational localization in glasses, Phys. Rev. B 43, 5039 (1991).

[20] V. L. Gurevich, D. A. Parshin, and H. R. Schober, Anharmonicity, vibrational instability, and the Boson peak in glasses, Phys. Rev. B 67, 094203 (2003).

[21] V. Martín-Mayor, G. Parisi, and P. Verrocchio, Dynamical structure factor in disordered systems, Phys. Rev. E 62, 2373 (2000).

[22] S. N. Taraskin, Y. L. Loh, G. Natarajan, and S. R. Elliott, Origin of the Boson Peak in Systems with Lattice Disorder, Phys. Rev. Lett. 86, 1255 (2001).

[23] W. Schirmacher, G. Ruocco, and T. Scopigno, Acoustic Attenuation in Glasses and Its Relation with the Boson Peak, Phys. Rev. Lett. 98, 025501 (2007).

[24] W. Schirmacher, The boson peak, Phys. Status Solidi B 250, 937 (2013).

[25] W. Schirmacher, Theory of vibrational anomalies in glasses, J. Noncryst. Sol. 407, 133 (2014).

[26] A. I. Chumakov, G. Monaco, A. Monaco, W. A. Crichton, A. Bosak, R. Rüffer, A. Meyer, F. Kargl, L. Comez, D. Fioretto, H. Giefers, S. Roitsch, G. Wortmann, M. H. Manghnani, A. Hushur, Q. Williams, J. Balogh, K. Parliński, P. Jochym, and P. Piekarz, Equivalence of the Boson Peak in Glasses to the Transverse Acoustic Van Hove Singularity in Crystals, Phys. Rev. Lett. 106, 225501 (2011).

[27] A. I. Chumakov, G. Monaco, A. Fontana, A. Bosak, R. P. Hermann, D. Bessas, B. Wehinger, W. A. Crichton, M. Krisch, R. Rüffer, G. Baldi, G. Carini, Jr., G. Carini, G. D’Angelo, E. Gilioli, G. Tripodo, M. Zanatta, B. Winkler, V. Milman, K. Refson, M.T. Dove, N. Dubrovinskaia, L. Dubrovinsky, R. Keding, and Y.Z. Yue, Role of Disorder in the Thermodynamics and Atomic Dynamics of Glasses, Phys. Rev. Lett. 112, 025502 (2014).

[28] R. Zorn, The boson peak demystified? Physics 4, 44 (2011).

[29] Y. Wang, L. Hong, Y. Wang, W. Schirmacher, and J. Zhang, Disentangling boson peaks and Van Hove singularities in a model glass, Phys. Rev. B 98, 174207 (2018).

[30] K. Watanabe and H. Tanaka, Direct Observation of MediumRange Crystalline Order in Granular Liquids Near the Glass Transition, Phys. Rev. Lett. 100, 158002 (2008).

[31] C. Brito, O. Dauchot, G. Biroli, and J. P. Bouchaud, Elementary excitation modes in a granular glass above jamming, Soft Matter 6, 3013 (2010).

[32] L. Zhang, J. Zheng, Y. Wang, L. Zhang, Z. Jin, L. Hong, Y. Wang, and J. Zhang, Experimental studies of vibrational modes in a two-dimensional amorphous solid, Nat. Commun. 8, 67 (2017).
[33] A. S. Keys, A. R. Abate, S. C. Glotzer, and D. J. Durian, Measurement of growing dynamical length scales and prediction of the jamming transition in a granular material, Nat. Phys. 3, 260 (2007).

[34] P. Charbonneau, J. Kurchan, G. Parisi, P. Urbani, and F. Zamponi, Fractal free energy landscapes in structural glasses, Nat. Commun. 5, 3725 (2014).

[35] P. Charbonneau, J. Kurchan, G. Parisi, P. Urbani, and F. Zamponi, Glass and jamming transitions: From exact results to finite-dimensional descriptions, Annu. Rev. Condens. Matter Phys. 8, 265 (2017).

[36] M. Wyart, L. E. Silbert, S. R. Nagel, and T. A. Witten, Effects of compression on the vibrational modes of marginally jammed solids, Phys. Rev. E 72, 051306 (2005).

[37] N. Xu, V. Vitelli, M. Wyart, A. J. Liu, and S. R. Nagel, Energy Transport in Jammed Sphere Packings, Phys. Rev. Lett. 102, 038001 (2009).

[38] L. Zhang, Y. Wang, J. Zheng, A. Sun, X. Sun, Y. Wang, W. Schirmacher, and J. Zhang, Level statistics and Anderson delocalization in two-dimensional granular materials, Phys. Rev. B 103, 104201 (2021).

[39] L. Wang, G. Szamel, and E. Flenner, Low-frequency excess vibrational modes in two-dimensional glasses, arXiv:2107.01505.

[40] H. Ikeda, Universal non-mean-field scaling in the density of states of amorphous solids, Phys. Rev. E 99, 050901(R) (2019).

[41] E. Stanifer, P. K. Morse, A. A. Middleton, and M. L. Manning, Simple random matrix model for the vibrational spectrum of structural glasses, Phys. Rev. E 98, 042908 (2018).

[42] E. Bouchbinder, E. Lerner, C. Rainone, P. Urbani, and F. Zamponi, Low-frequency vibrational spectrum of mean-field disordered systems, Phys. Rev. B 103, 174202 (2021).

[43] E. Degiuli, A. Laversanne-Finot, G. Düring, E. Lerner, and M. Wyart, Effects of coordination and pressure on sound attenuation, boson peak and elasticity in amorphous solids, Soft Matter 10, 5628 (2014).

[44] S. Franz, G. Parisi, P. Urbani, and F. Zamponi, Universal spectrum of normal modes in low-temperature glasses, Proc. Natl. Acad. Sci. USA 112, 14539 (2015).

[45] M. Alam and S. Luding, First normal stress difference and crystallization in a dense sheared granular fluid, Phys. Fluids 15, 2298 (2003).

[46] M. Alam, P. Shukla, and S. Luding, Universality of shearbanding instability and crystallization in sheared granular fluids, J. Fluid Mech. 615, 293 (2008)

[47] B. P. Tighe, A. R. van Eerd, and T. J. Vlugt, Entropy Maximization in the Force Network Ensemble for Granular Solids, Phys. Rev. Lett. 100, 238001 (2008).

[48] D. Kaya, N. L. Green, C. E. Maloney, and M. F. Islam, Normal modes and density of states of disordered colloidal solids, Science 329, 656 (2010).

[49] T. S. Majmudar and R. P. Behringer, Contact force measurements and stress-induced anisotropy in granular materials, Nature (London) 435, 1079 (2005).

[50] J. F. Peters, M. Muthuswamy, J. Wibowo, and A. Tordesillas, Characterization of force chains in granular material, Phys. Rev. E 72, 041307 (2005)

[51] K. Binder and W. Kob, Glassy Materials and Disordered Solids (World Scientific, Singapore, 2011), p. 55. 
[52] K. Chen, T. Still, S. Schoenholz, K. B. Aptowicz, M. Schindler, A. C. Maggs, A. J. Liu, and A. G. Yodh, Phonons in twodimensional soft colloidal crystals, Phys. Rev. E 88, 022315 (2013).

[53] H. Tanaka, T. Kawasaki, H. Shintani, and K. Watanabe, Criticallike behaviour of glass-forming liquids, Nat. Mater. 9, 324 (2010).

[54] D. Bi, J. Zhang, B. Chakraborty, and R. P. Behringer, Jamming by shear, Nature (London) 480, 355 (2011).

[55] T. S. Majmudar, M. Sperl, S. Luding, and R. P. Behringer, Jamming Transition in Granular Systems, Phys. Rev. Lett. 98, 058001 (2007).

[56] G. J. Gao, J. Bławzdziewicz, and C. S. O’Hern, Frequency distribution of mechanically stable disk packings, Phys. Rev. E 74, 061304 (2006).

[57] P. W. Anderson, Absence of diffusion in certain random lattices, Phys. Rev. 109, 1492 (1958).

[58] H. Hu, A. Strybulevych, J. H. Page, S. E. Skipetrov, and B. A. van Tiggelen, Localization of ultrasound in a three-dimensional elastic network, Nat. Phys. 4, 945 (2008).

[59] B. J. Huang and T. M. Wu, Localization-delocalization transition in Hessian matrices of topologically disordered systems, Phys. Rev. E 79, 041105 (2009).
[60] S. D. Pinski, W. Schirmacher, T. Whall, and R. A. Römer, Localization-delocalization transition for disordered cubic harmonic lattices, J. Phys.: Condens. Matter 24, 405401 (2012).

[61] S. D. Pinski, W. Schirmacher, and R. A. Römer, Anderson universality in a model of disordered phonons, Europhys. Lett. 97, 16007 (2012).

[62] H. Tong, P. Tan, and N. Xu, From crystals to disordered crystals: A hidden order-disorder transition, Sci. Rep. 5, 15378 (2015).

[63] Y. Nie, H. Tong, J. Liu, M. Zu, and N. Xu, Role of disorder in determining the vibrational properties of mass-spring networks, Front. Phys. 12, 126301 (2017).

[64] H. Mizuno, L. E. Silbert, and M. Sperl, Spatial Distributions of Local Elastic Moduli Near the Jamming Transition, Phys. Rev. Lett. 116, 068302 (2016).

[65] See Supplemental Material at http://link.aps.org/supplemental/ 10.1103/PhysRevResearch.3.L032067 for the additional information on the definition of disorder parameter $\eta$, the procedure to calculate the local moduli, the spatial autocorrelations of modulus fluctuations, the fluctuations of the modulus for different coarse-graining size, the fluctuations of the modulus when $w=2$, and the joint influence of $\delta G /\langle G\rangle$ and $\eta$ on the shape of the boson peak 